\title{
O Uso de Tirinhas como Objeto de Aprendizagem (OA) na Formação Continuada de Professores
}

\author{
Darkson Fernandes da Costa ${ }^{1}$, Arianny de Sousa Lira' ${ }^{1}$ João Batista da Silva ${ }^{2}$, \\ Juscileide Braga de Castro ${ }^{13}$, Gilvandenys Leite Sales ${ }^{1}$
}

${ }^{1}$ Programa de Pós-graduação em Ensino de Ciências e Matemática (PGECM) - Instituto Federal do Ceará (IFCE) - Caixa Postal: 60040-215 - Fortaleza - CE - Brasil

${ }^{2}$ Programa de Pós-graduação em Educação (PPGE) - Universidade Estadual do Ceará (UECE) - Caixa Postal 60741-000 - Fortaleza - CE - Brasil

${ }^{3}$ Faculdade de Educação (FACED) - Universidade Federal do Ceará (UFC) - Caixa Postal: 60020-110 - Fortaleza - CE - Brasil

darkson@gmail.com, \{arianny_sousa,joaobathista82\}@hotmail.com, juscileide@virtual.ufc.br, denyssales@ifce.edu.br

\begin{abstract}
This work arose during a workshop given to the undergraduates who were participating in the Active Methodologies Day, 2019. The objective was to present a proposal for the use of comic strips as a Learning Object (LO), linked to a methodological teaching path model. In addition, the free HQ editing website Toondoo.com was used. The results demonstrated an evolution in the understanding of the technological tool during the workshop by the teachers. However, there is a need to use teaching methodologies which use comic strips to investigate their effects on students learning in each of the teaching areas and in accordance with some methodological steps proposed in the literature.
\end{abstract}

Resumo. Este trabalho surgiu durante uma oficina ministrada à licenciandos na Jornada de Metodologias Ativas 2019. O objetivo foi apresentar uma proposta de utilização de tirinhas como Objeto de Aprendizagem (OA), vinculada à um modelo de percurso metodológico de ensino. Além disso, foi utilizado o site gratuito de edição de HQ Toondoo.com. Os resultados demonstraram uma evolução na compreensão da ferramenta tecnológica no decorrer da oficina pelos professores. Entretanto, há uma necessidade de se empregar metodologias de ensino que utilizem tirinhas para investigar os seus efeitos na aprendizagem dos alunos em cada uma das áreas de ensino e em conformidade com alguns passos metodológicos propostos na literatura.

\section{Introdução}

A democratização do acesso à internet em dispositivos móveis contribui para o acesso à informação. Muitas instituições de ensino oferecem aos alunos e aos professores ferramentas que facilitam o ensino e a aprendizagem. Segundo Hino et al. (2019) os alunos que utilizam computadores com mais frequência apresentam melhores resultados na aprendizagem em comparação com os alunos que usam raramente. Ademais, grande parte dos jovens encontram-se imersos em um meio repleto de Tecnologias Digitais de Informação e Comunicação (TDIC) desde cedo, seja direcionado para o entretenimento ou para a comunicação [Lacerda et al. 2017]. 
As escolas não devem ignorar as mudanças que estão ocorrendo com os jovens contemporâneos. Nesse sentido, a formação continuada de professores vem apresentando um papel fundamental nessa atualização curricular do ensino, para favorecer o uso de tecnologias, de metodologias e de abordagens que coloquem os alunos como ativos na construção do seu próprio conhecimento.

Boa parte dos professores buscam formas de manter seus alunos envolvidos e engajados com as propostas metodológicas das aulas. No entanto, muitos estão acostumados com o método tradicional de ensino, baseados na ideia de que a maioria das TDIC pode diminuir a atenção dos alunos e até mesmo dificultar o andamento das aulas por estarem conectados em um mundo virtual, fora da sala [Moreira 2017]. O computador tornou-se parte inseparável do ambiente escolar e exige uma reorganização para uma adesão dessas tecnologias em sala de aula [Hino et al. 2019].

Para que os professores possam usufruir das novas competências técnicas, cognitivas e sociais proporcionadas por essas ferramentas é necessário que seja alfabetizado na linguagem digital [Hino et al. 2019]. Essas questões ganharam maiores proporções a partir da Base Nacional Comum Curricular (BNCC), documento norteador do currículo brasileiro, que indica em uma de suas competências gerais a cultura digital, sugerindo que as TDIC sejam não apenas utilizadas, mas também construídas de forma ética, significativa e crítica, considerando as diferentes práticas sociais e, portanto, escolares [Brasil 2017].

Torna-se importante citar que não basta identificar as estratégias de ensino que podem ser utilizadas com as tecnologias como ferramenta. O professor precisa compreender as possibilidades pedagógicas das ferramentas que pretende trabalhar [Novais 2010]. Nessa perspectiva, o uso de tecnologias no ensino deve ser dominado pelo professor para explorar as capacidades dos alunos de manuseá-las por conta própria e proporcionar um ambiente de descobertas autênticas.

Em uma pesquisa realizada por Teles et al. (2020) ao analisar a implementação de TDIC em sala de aula por licenciados, foi identificado evidências de que as tecnologias foram inseridas em sala de aula com o intuito de informatizar o processo educacional, sem a modificação ou a adaptação de bases metodológicas. Nessa perspectiva, foram encontradas dificuldades pelos licenciados para se gerar um percurso metodológico com o uso de TDIC, em que o aluno torne-se protagonista, no sentido de produzir seus próprios conhecimentos [Teles et al. 2020].

Segundo Costa et al. (2019), somente implementar as TDIC e qualquer outra ferramenta facilitadora de aprendizagem em sala de aula, não se caracteriza como fator principal para se provocar uma mudança significativa no ensino. É preciso que as ferramentas sejam implementadas com um propósito adequado para se favorecer a autonomia do aluno e o pensar criticamente [Costa et al. 2019].

Uma forma de favorecer a autonomia e o engajamento dos estudantes é explorar atividades e materiais significativos que sejam populares entre o público alvo, como por exemplo, as Histórias em Quadrinhos (HQs). Quando falamos em HQs, a primeira coisa que vem à mente são as produções editoriais em jornais e revistas nos seus mais variados formatos. As tirinhas, por exemplo, são mais popularmente usadas em jornais impressos. Geralmente em formato horizontal, apresenta uma história fechada de no máximo cinco quadros, rápida e com um intuito de passar informação, que algumas vezes podem ser bem humoradas. 
Segundo Rama e Vergueiro (2008) as HQs, juntamente com o cinema, constituem dois meios de comunicação muito importante no século XX. Com isso, as HQs e as tirinhas podem se tornar uma importante estratégia para os professores interagirem com os alunos por meio das linguagens que eles já adquiriram familiaridade, considerando seus conhecimentos prévios do cotidiano.

Há pouco, as tirinhas eram vistas apenas como entretenimento e diversão. Hoje, as tirinhas têm um forte papel na construção de opiniões, influenciando diretamente o campo da Educação como recurso pedagógico [Lacerda et al. 2017]. As tirinhas passaram a incorporar livros didáticos e até mesmo provas de vestibulares como um auxílio na forma de apresentar um assunto ou como uma crítica à diversos temas da sociedade. Além da rapidez para se passar uma informação para os leitores, as tirinhas conseguem fazer as pessoas refletirem sobre o que foi abordado no assunto, que muitas vezes são temas polêmicos da atualidade.

Diante do que foi justificado, o presente trabalho tem como objetivo foi apresentar uma proposta de utilização de tirinhas como Objeto de Aprendizagem (OA), vinculada à um modelo de percurso metodológico de ensino.

Para implementar as tirinhas no ensino foi utilizado o editor de quadrinhos Toondoo ${ }^{1}$. Uma ferramenta gratuita de fácil manuseio, voltado para pessoas que desejam criar, editar e compartilhar quadrinhos ou desenvolver seus próprios personagens e suas narrativas em uma plataforma online.

A presente pesquisa encontra-se dividida em seções. A seção 2 apresenta a fundamentação teórica da pesquisa, elencando os princípios que norteiam o uso das tirinhas como OA e o site utilizado para a edição de tirinhas (Toondoo.com). Na seção 3 será apresentado as etapas metodológicas de aplicação das tirinhas na oficina para os professores. Em seguida, na seção 4, encontram-se os resultados da pesquisa e uma breve análise das produções dos professores. Por último, na seção 5, apresentam-se as considerações finais.

\section{Objetos de Aprendizagem (OA)}

Atualmente, existem diferentes descrições e definições para um OA, apresentado por diferentes autores na literatura. Segundo Wiley (2002), OA pode ser qualquer recurso digital que poderá ser reutilizado para a aprendizagem. Isto inclui animações, imagens, gifs, vídeos, páginas na Web etc. Com base em Nash (2005) os OA são como blocos de informações que encontram-se à disposição dos professores para que os conectem da maneira que identificarem ser mais eficientes para a aprendizagem dos alunos.

Segundo Castro-Filho (2007) e Sales (2005) os OA possuem as seguintes características: a) Podem ser digitais, o uso de OA deve ser utilizado de preferência pela internet para que seja amplamente reproduzido e reutilizado; b) Ser pequeno, a interação com o OA deve ser rápida e intuitiva em que o aluno possa aprender no tempo de uma ou duas aulas; e c) Conter um objetivo bem esclarecido, ou seja, no final da interação com o OA proposto pelo professor, o aluno seja capaz de ampliar/melhorar seus conhecimentos e/ou habilidades.

\footnotetext{
${ }^{1}$ Toondoo refere-se ao site de edição de quadrinhos [http://www.Toondoo.com/]. Atualmente o site, Toondoo, encontra-se fora de funcionamento. No entanto, existem sites/ferramentas que pode suprir as mesmas características do Toondoo como exemplo o [https://www.makebeliefscomix.com/].
} 
Para Wiley (2002), o OA encontra-se diretamente relacionado com o meio digital, diferentemente de Nash (2005) que apresenta o OA sem possuir vínculo com recursos tecnológicos. Com Nash (2005) a categorização de OA torna-se mais ampla ao incluir os recursos analógicos. Nessa perspectiva, usaremos para o presente trabalho uma descrição de OA mais ampla, com base em Castro-Filho (2007), Sales (2005), Wiley (2002) e Nash (2005). Assim sendo, o OA pode ser qualquer instrumento, digital e não digital que poderá ser utilizado, reutilizado ou referenciado dentro de um percurso metodológico de aprendizagem.

Considerando o contexto de produção de OA por professores em formação, Castro et al. (2017) desenvolveram um projeto de produção de materiais digitais como parte da disciplina de Ensino de Matemática ofertada pela Universidade Federal do Ceará. Dentre os resultados, os pesquisadores apontam que a abordagem construcionista adotada no projeto, de aprender fazendo, contribuiu para a compreensão conceitual, teórica e metodológica dos conteúdos trabalhados, favorecendo a formação dos futuros professores.

Ao analisar a pesquisa de Castro et al. (2017) podemos evidenciar a produção para a construção de conhecimento, assim como na perspectiva de propiciar que docentes possam produzir OA para o ensino. Nesta mesma perspectiva, Lira, Leitão e Castro (2019) propuseram uma oficina com 14 professores que atuam em diferentes níveis. A partir das análises realizadas, as pesquisadoras destacam a importância de possibilitar ao professor o papel de autor de seu próprio material didático, fazendo-os perceber 0 processo vivenciado como uma metodologia a ser desenvolvida na escola.

Destarte, sobre a importância da produção de materiais didáticos, Lacerda (2017) contribuiu para a formação continuada de professores ao apresentar o Toondoo como recurso pedagógico, bem como reforçou a importância da utilização de TDIC no ambiente escolar, em que o educador pôde aproximar o conteúdo à realidade dos estudantes. Nesse viés, a produção de tirinhas foi proposta como estratégia para o ensino e a aprendizagem de conceitos, podendo ser utilizada em diferentes áreas e de forma interdisciplinar.

As tirinhas podem ser utilizadas como OA, pois incorporam as características que compõem as descrições de um OA apresentadas por Castro-Filho (2007) e Sales (2005). Logo, possuem as seguintes características: a) As tirinhas possuem um amplo alcance dentro dos meios digitais ao ser utilizadas em redes sociais como memes ou formas de se abordar conceitos; b) Geralmente as tirinhas possuem uma narrativa rápida, em que o enredo da história ocorre em no máximo cinco quadros, com introdução, desenvolvimento e conclusão; c) As tirinhas possuem um objetivo bem específico para a conclusão do seu enredo. Ademais, as tirinhas podem apresentar características direcionadas para o humor, com isso, tornam-se cada vez mais contextualizadas para o aluno. Neste sentido, na próxima seção, discorre-se sobre a ferramenta de edição de tirinhas, Toondoo.

\subsection{Editor de tirinhas - Toondoo}

Para o uso de tirinhas em sala de aula usamos a ferramenta/site de edição, Toondoo.com. Com o site, o professor pode utilizar as tirinhas produzidas em todos os momentos da sua aula, como atividade de casa, pré e pós-teste ou para abordar um determinado conteúdo. Nesse sentido, as tirinhas podem funcionar como forma avaliativa e como uma possibilidade para se abordar conceitos. 
O Toondoo como ferramenta de edição e de desenvolvimento de tirinhas, apresenta em sua interface elementos integrantes e fundamentais para construção de quadrinhos, como: personagens, cenários, objetos e balões. Para se inserir qualquer elemento, basta clicar no que se deseja incluir e arrastar para dentro do cenário (Figura 1). Esses elementos incluídos nos cenários podem ser aumentados/diminuídos, clonados e até as expressões faciais de cada personagem podem ser modificadas. Isso possibilita que cada tirinha tenha características únicas.

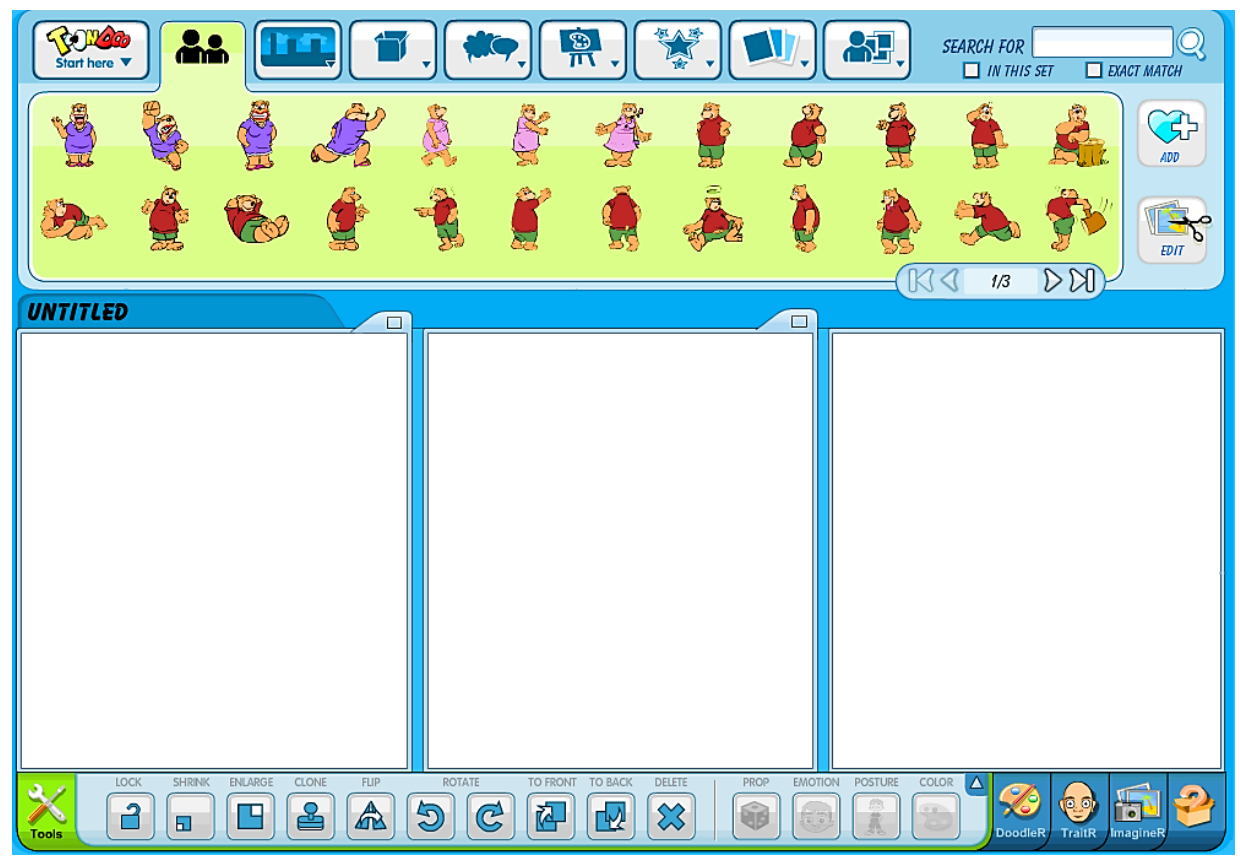

Figura 1 - Interface de edição de tirinhas do Toondoo

Quando o aluno desenvolve tirinhas, ele produz um artefato, logo, formula conceitos, desenvolve o raciocínio e se torna capaz de transmitir o que ele domina e sabe sobre determinado assunto [Castro et al 2017; Lira, Leitão e Castro 2019]. O uso e a produção de tirinhas pelo professor em sala de aula direcionam-no a uma cultura digital, em que ele pode ser autor de seu próprio material didático, assim, como compreende diferentes abordagens metodológicas em que a tirinha é um OA que faz parte do cotidiano dos alunos.

A seguir serão abordados os detalhes metodológicos da aplicação para a formação de professores.

\section{Percurso Metodológico de Ensino}

O percurso metodológico foi realizado durante uma oficina para cinco professores de áreas distintas, no decorrer da Jornada de Metodologias Ativas no ano de 2019. Esta jornada foi realizada com apoio do projeto Ciências, Artes e Tecnologia (CIARTEC) do Instituto Federal de Educação, Ciência e Tecnologia do Ceará (IFCE), campus de Fortaleza. A Jornada de Metodologias Ativas é uma capacitação que difunde o uso de metodologias centradas no aluno. Além disso, orienta e sugere práticas pedagógicas e o uso e a produção de ferramentas digitais. A oficina teve como intuito favorecer 0 manuseio da ferramenta Toondoo para os professores, assim como reflexões sobre a ferramenta e possíveis estratégias metodológicas para se utilizar em sala de aula. 
Trata-se de um estudo exploratório, com dados qualitativos. A aplicação do percurso metodológico contou com dois momentos para a exploração da ferramenta e dois momentos explicativos. Para um delineamento mais detalhado, usou-se os passos: a) Contrato didático e informações da ferramenta; b) Interação com a ferramenta; c) Situação conflitiva no ensino usada como exemplo; e d) Nova interação com a ferramenta.

\section{a) Contrato didático e informações da ferramenta}

Nessa etapa foi evidenciada a importância de se utilizar materiais manipulativos, tecnológicos e contextualizados com a realidade do aluno, como forma de enriquecer a práxis pedagógica. Ademais, foi apresentado os objetivos e os passos da aula, assim como o tema da situação conflitiva que seria utilizada como exemplo. Outro passo desta etapa foi apresentar a ferramenta Toondoo para os professores com os detalhes de manuseio, uso dos balões, dentre outros aspectos.

Os professores foram informados de que ao final da oficina, seriam capazes de: a) Manusear a ferramenta Toondoo sem muitas dificuldades; e b) Compreender uma proposta de percurso metodológico para o uso de tirinhas em sala de aula.

\section{b) Interação com a ferramenta}

Esta fase foi reservada para os participantes (professores em formação) manipularem a ferramenta e desenvolverem uma tirinha, conforme o assunto de sua predileção. A parte manipulativa da oficina caracteriza-se como uma das partes principais, é fato que o aluno aprende de forma mais significativa quando manipula e interage com o OA ou qualquer outra ferramenta usada no decorrer do ensino.

\section{c) Situação conflitiva no ensino usada como exemplo}

Na presente fase foram distribuídas situações conflitivas no ensino de Física. Estas incluíam os detalhes básicos das leis de Newton, sem a pretensão de se avaliar o que os professores dominavam sobre o conteúdo de Física abordado. Usamos o assunto referente a aplicação de uma força em um corpo, apenas como um exemplo para se aplicar a metodologia de produção de tirinhas. Após uma breve explicação do assunto os alunos desenvolveram suas tirinhas com base na apresentação.

Torna-se conveniente citar que nem todos os professores são da área da Física e o uso das situações conflitivas na Física foram apenas para se demonstrar aos professores uma proposta de uso de tirinhas em sala de aula e essa proposta deve ser adaptada conforme a área respectiva dos professores. Todos esses detalhes foram apresentados na primeira fase, que corresponde ao contrato didático do percurso metodológico utilizado.

\section{d) Nova interação com a ferramenta.}

Foi proposto pelo professor da oficina que fossem utilizados três cenários para o desenvolvimento das tirinhas, conforme o tema apresentado na oficina. O motivo é que as tirinhas sejam rápidas tanto para se desenvolver como para se compreender. Ademais, os três cenários propostos nas situações conflitivas seriam: introdução, desenvolvimento e conclusão; característica básica da maioria das formulações intelectuais.

A seguir será apresentado os resultados e as discussões, bem como algumas produções dos participantes apresentados como retorno da atividade na oficina. 


\section{Resultados e Discussões}

Para os resultados da pesquisa é importante destacar que o intuito era de familiarizar os participantes com a ferramenta de edição de quadrinhos Toondoo e não avaliar a compreensão dos conceitos de Física abordados durante a implementação. A figura 2 mostra um exemplo de produção realizada pelos participantes, que escolheram como tema a $3^{\text {a }}$ Lei de Newton.
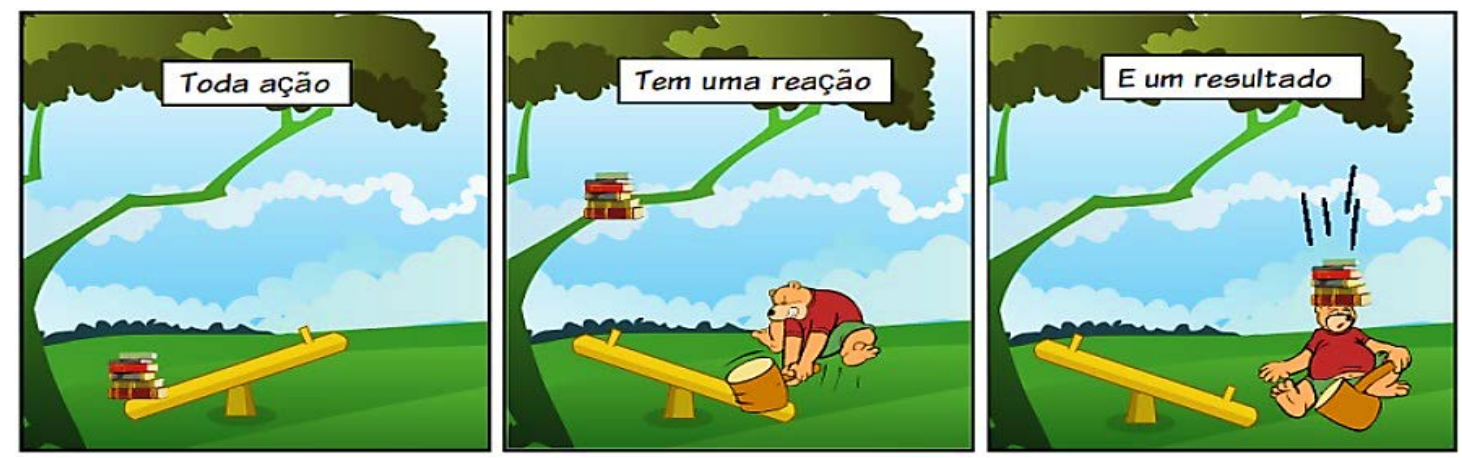

Figura 2 - Tirinha $3^{\text {a }}$ Lei de Newton: produção do participante

A tirinha $3^{\text {a }}$ Lei de Newton foi desenvolvida sobre a situação conflitiva proposta de forma livre, solicitada pelo professor da oficina, correspondente à etapa b) Interação com a ferramenta, dos passos metodológicos da oficina. Como desafio, foi requisitado na etapa: d) Nova interação com a ferramenta, a produção de uma tirinha que substituísse apenas o cenário de conclusão. A figura 3 mostra alguns modelos apresentados pelos participantes.
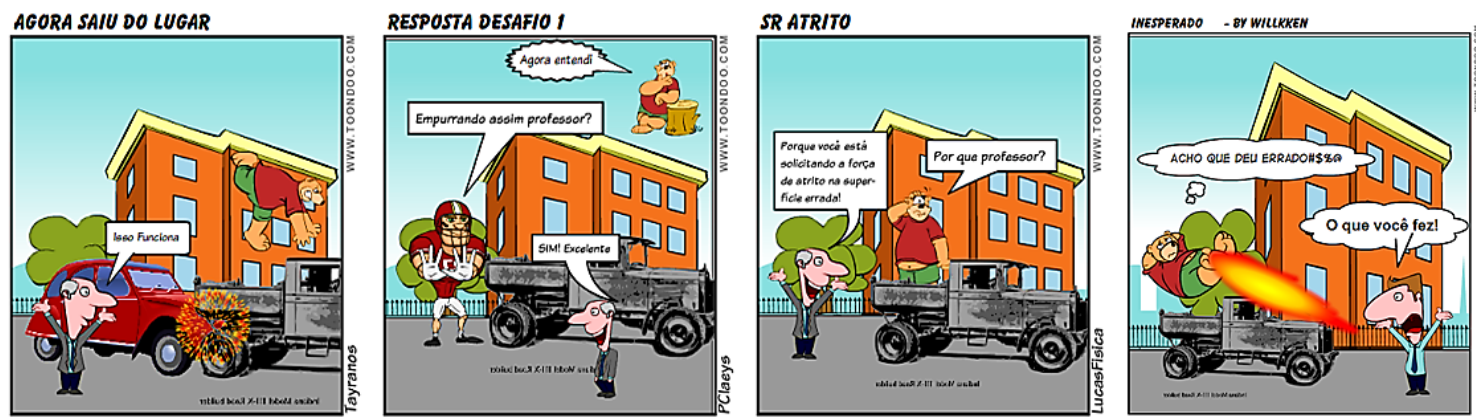

Figura 3 - Cenário conclusão: produção dos participantes

A implementação dessas atividades manipulativas foi importante para os professores conseguirem compreender o funcionamento da ferramenta Toondoo. Destacamos ainda que a participação como alunos na oficina possibilitou a compreensão dos detalhes de um percurso metodológico para o uso de tirinhas em sala de aula. Desta forma, fica mais evidente, bem como facilita a realização de uma possível intervenção em sala de aula.

Por se tratar de uma interface bem intuitiva e simples, os professores não tiveram tantas dificuldades para manuseá-las, deixando o desafio da atividade direcionado para compreender a metodologia empregada no uso de tirinhas em sala de aula. Os professores ainda se mostraram interessado com o tipo de metodologia que foi aplicado durante a atividade. 
Diante dos objetivos e dos resultados obtidos durante a pesquisa foi observado que os professores conseguiram compreender a ferramenta Toondoo e perceber, na prática, a aplicação de uma metodologia abordando conceitos de uma respectiva área. Além disso, nenhum professor se mostrou apático ou desinteressado com a aplicação da metodologia usando a ferramenta Toondoo, sendo que a atividade proposta foi realizada por todos os participantes da aplicação.

\section{Considerações Finais}

Observamos que o site Toondoo se mostrou útil para os professores que tinham dificuldades para desenhar com lápis e papel e expor seus conhecimentos por meio dos quadrinhos. Foi sugerido, durante a oficina, que fossem apresentados os quadrinhos como uma introdução ao conteúdo abordado na aula e como forma avaliativa para os professores saberem se os alunos compreenderam o que foi discutido em sala de aula.

Levando em consideração o objetivo proposto no início do trabalho: apresentar uma proposta de utilização de tirinhas como Objeto de Aprendizagem (OA), vinculada à um modelo de percurso metodológico de ensino; os professores se mostraram interessados em: utilizar as TDIC em suas práticas pedagógicas; explorar as tirinhas como um OA, com potencial para o ensino; além de vislumbrar a metodologia empregada como relevante para cumprir esse objetivo.

Os resultados obtidos no presente trabalho sugerem que sejam abordadas metodologias centradas no aluno, pois possibilitam o raciocínio crítico dos estudantes em sala de aula. Metodologias ativas como: instrução por pares, sala de aula invertida, aprendizagem baseada em problemas ou projetos, podem ser utilizadas com as TDIC, permitindo o caminho para uma educação significativa. Para trabalhos futuros, pretendemos ofertar minicursos e formações destinadas aos professores.

\section{Referências}

Arantes, A. R.; Miranda, M. S.; Studart, N. (2010). “Objetos de aprendizagem no ensino de física: usando simulações do PhET”. Física na Escola, 11(1), 27-31. Disponível em: http://www1.fisica.org.br/fne/edicoes/category/9-volume-11-n-1abril?download=62:objetos-de-aprendizagem-no-ensino-de-fisica-usandosimulacoes-do-phet

Brasil. (2017) Ministério da Educação. “Base Nacional Comum Curricular- BNCC”. Brasília.

Castro-Filho, J. A. (2007). "Objetos de aprendizagem e sua utilização no ensino de matemática”. In: Encontro Nacional de Educação Matemática, 9, 2007, Belo Horizonte. Anais... Belo Horizonte, MG : SBEM - Sociedade Brasileira de Educação Matemática. Disponível em: http://paginapessoal.utfpr.edu.br/kalinke/grupos-depesquisa/novas-tecnologias/grupos-depesquisa/pde/pdf/objetos_de_aprendizagem_e_EM.pdf

Castro, J. B.; Soares, I. O.; Vianna, E. P. N.; Sousa, J. S. (2017). “Lógica Criativa: a construção de mídias digitais para o ensino de matemática”. In: Anais dos Workshops do VI Congresso Brasileiro de Informática na Educação (WCBIE 2017). VI Congresso Brasileiro de Informática na Educação (CBIE 2017). Disponível em: http://dx.doi.org/10.5753/cbie.wcbie.2017.485 
Costa, D. F.; Monteiro, J. A.; Castro, J. B.; Coutinho-Júnior, A. L.; Sales, G. L. (2019). "Strategies for the elaboration of a gamed activity script". Research, Society and Development, 8(11), 188111451. Disponível em: https://dialnet.unirioja.es/servlet/articulo?codigo=7164825

Hino, M. C.; Przeybilovicz, E.; Coelho, T. R. (2019). "Bring your own device (BYOD): entendendo uma nova prática no ambiente acadêmico”. Acta Scientiarum. Education, 41, e42661-e42661. Disponível em: http://periodicos.uem.br/ojs/index.php/ActaSciEduc/article/view/42661

Lacerda, C. C.; Sepel, L. M. N.; Falkembach, G. M. (2017). “Toondoo: o uso de histórias em quadrinhos como objeto de aprendizagem na formação continuada de professores”. Imagens da educação, 7(3), 63-73. Disponível em: http://periodicos.uem.br/ojs/index.php/ImagensEduc/article/view/38294

Lira, A. S.; Leitão, D. A.; Castro, J. B. (2019). "Como o Processo de Produção de Mídias pode contribuir para a Formação docente?”. RENOTE. Revista Novas Tecnologias na Educação, v. 17, p. 425-434, 2019. Disponível em: https://www.seer.ufrgs.br/renote/article/view/95850

Moreira, M. A. (2017). "Grandes desafios para o ensino da Física na educação contemporânea”. Revista Do Professor de Física, 1(1), 1-13. Disponível em: https://periodicos.unb.br/index.php/rpf/article/view/7074

Nash, S. (2005). "Learning objects, learning object repositories, and learning theory: Preliminary best practices for online courses”. Interdisciplinary Journal of E-Learning and Learning Objects, 1(1), 217-228. Disponível em: https://www.learntechlib.org/p/44877/

Novais, A. E. (2010). "Experiências genuinamente digitais e a herança do impresso: o que ajuda na interação com as interfaces gráficas”. RIBEIRO, AE et Al. Linguagem Tecnologia e Educação. São Paulo: Peirópolis.

Rama, A.; Vergueiro, W. (2008). “Como usar as histórias em quadrinhos na sala de aula”. Editora Contexto. São Paulo.

Sales, G. L. (2005). “QUANTUM: Um Software para Aprendizagem dos Conceitos da Física Moderna e Contemporânea”. Dissertação de Mestrado, CEFET-CE. Disponível em: http://bdtd.ibict.br/vufind/Record/UECE$\underline{0}$ 56424d765dbfb08a0c67972e8638496c

Teles, G.; Soares, D. M. R.; de Lima, L.; Loureiro, R. C. (2020). “Docência e Tecnologias Digitais na Formação de Professores: Planejamento e Execução de Aulas por Licenciandos”. Brazilian Journal of Technology, 3(2), 73-84.

Wiley, D. A. (2002). “The instructional use of learning objects” (Vol. 1). Bloomington, IN: Agency for instructional technology. 